\title{
ON THE LOGIC UNDERLYING COMMON SENSE
}

\author{
Janos J. Sarbo \\ Institute for Computing and Information Sciences Radboud University, Nijmegen, The Netherlands, janos@cs.ru.nl
}

Keywords: common sense, naive logic, knowledge representation, signs, cognitive activity, Peirce, semiotics

\begin{abstract}
In order to endow computers with common sense with respect to specific domains we need to have a representation of the world and make commitments about what knowledge is and how it is obtained. This paper is an attempt to introduce such a representation and underlying 'naive' logic on the basis of an analysis of the properties of cognitive activity. This paper is of interest to those engaged in the development of user interfaces and ontologies, as well as to those interested in the semiotic aspects of problem specification and requirement engineering. The focus of this paper is on the theory, applications are briefly mentioned due to lack of space.
\end{abstract}

\section{Introduction}

According to a seminal paper by McCarthy and Hayes (McCarthy and Hayes, 1969) "a computer program capable of acting intelligently in the world must have a general representation of the world in terms of which its inputs are interpreted. Designing such a program requires commitments about what knowledge is and how it is obtained." The assumption taken by this paper is that the world consists of phenomena that are interactions and that knowledge arises from the observation of such phenomena by means of signs. An interaction may occur between entities, also called qualities, that are in principle independent. An observation is a re-presentation of an interaction between an external quality (input stimulus) and the observer, in which both entities are interpreted as signs.

This representation by the observer, as an interpreting system, has a 'goal', which is the (re)cognition of the input stimulus as meaningful. For example, the interaction between a piece of wood and sufficient energy (qualities), appearing as burning (phenomenon), can be signified by the visual sign of the arising smoke and fire. This sign, as a quality, may interact with the eyes, and that event, as a sign, may be recognized by the brain/mind as a representation of the burning phenomenon.

The observation of complex phenomena may re- quire the representation of a series of interaction events. As events governed by a goal are a process, and, following our assumption, qualities and events are interpreted as signs, we conclude that observations and knowledge must be a process of sign interactions.

Human observation is a product of cognitive activity by the brain/mind (Kalat, 2004), (Gazzaniga, 1998). This paper is an attempt to show that on the basis of an analysis of the properties of cognitive activity a representation for knowledge can be introduced. Such a representation and the underlying logical interpretation is what I shall call common sense and 'naive' logic. Other important elements of common sense, such as intelligence and learning are beyond the scope of this work.

According to Aristotle, the common sense (sensus communis) is the hart where all information from different senses are bound into an intelligible whole. Following the common sensist, it is the sum of original principles found in normal minds (Bergman, 2000). In the popular interpretation (MerriamWebster), it is a sound and prudent judgment based on a simple perception of the situation or facts.

In this paper I take the popular view as my starting point, skip the qualifications, "sound and prudent", and concentrate on the machinery implied: How do we account for the relation between the simple perception and the judgment? What is a simple percep- 
tion? How it is transformed into a judgment and what is involved? To this end, I assume that common sense is the brain's potential for a process (re)cognizing the 'naive' or common sense meaning of the input stimulus, as a sign. That potential and that type of process must be common to all human interpreter, as it is unlikely that cognitive activity as a process would be different in each human and, yet, common concepts can arise.

\section{A model of cognitive activity}

The duality involved in all phenomena is the key to their interpretation as meaningful. For example, in the observation of burning, the visual stimulus (first quality) may trigger the eyes (second quality). This interaction can be represented by the eyes, by means of comparing the activation of the receptors triggered by the current input, with the activation of those that are not activated (the activation of the second type of receptors may represent the background of the observation). The result of this comparison, a bio-electric signal, is capable of re-presenting the input stimulus (and, transitively so, the burning phenomenon) as a change with respect to some earlier state. This bioelectric signal may trigger the memory, and that interaction may be recognized by the brain (possibly through a sequence of re-presentation events) in a meaningful reaction such as shouting 'Fire!' or running away.

On the basis of this example, a model of cognitive activity, as a process, can be defined as follows. In an observation the external stimulus (effect) is effecting the brain appearing as a state. This interaction is re-presented by the brain through sampling the sensory input in a percept (a percept is a collection of qualia; qualities as perceived are called a quale in cognitive theory (Harnad, 1987)). The current percept is compared with the previous one, and this enables the brain to distinguish between two sorts of input qualia (in short, input): one, which was there and remained there, which can be called a 'state'; and another, which, though it was not there, is there now, which can be called an 'effect'.

Let me emphasize that any quality can be interpreted as a state or an effect. Also, that there may be any number of qualities involved in an interaction, but according to the theory of this paper those qualities are always distinguished by cognitive activity in two collections, state and effect, that are treated as single entities.

\subsection{The processing schema}

The interaction between the state and effect qualia appears as an event. Each occurrence of such an event triggers the memory, generating a response. This memory response is a representation of the brain's 'knowledge', as an interpreting system, about the properties of the external stimulus. Such properties, that are learned in earlier observations, are an expression of the brain's potential for combining with a type of input effect, depending on the brain's actual state. The total of those properties is called the context of the observation, containing all possible combinatory properties associated with the input event.

The primary task of cognitive processing is the interpretation of the external stimulus, in the light of its context. Following the theory of this paper, first presented in (Farkas and Sarbo, 2000), I assume that the input of cognitive processing consists of two types of qualia, appearing as a 'primordial soup' $\left(\left[\begin{array}{ll}q_{1} & q_{2}\end{array}\right]\right)$. The stages of the recognition process can be defined as follows (see also fig. 1). I use the convention that square brackets indicate that an entity is not yet interpreted as a sign, and no bracketing or the usual bracket symbols, if some interpretation is already available.

(1) sorting: $\left[q_{1}\right],\left[q_{2}\right]$ the identification of the two types of qualia in the 'primordial soup';

(2) abstraction: $q_{1}, q_{2}$ the separation of the collections of the two types of qualia;

(3) complementation: $\left(\mathrm{q}_{1}, \mathrm{C}\right),\left(\mathrm{q}_{2}, \mathrm{C}\right)$ the linking of the qualia with their combinatory properties ([C]);

(4) predication: $\left(\mathrm{q}_{1}, \mathrm{C}\right)-\left(\mathrm{q}_{2}, \mathrm{C}\right)$ the establishment of a relation between the completed qualia.

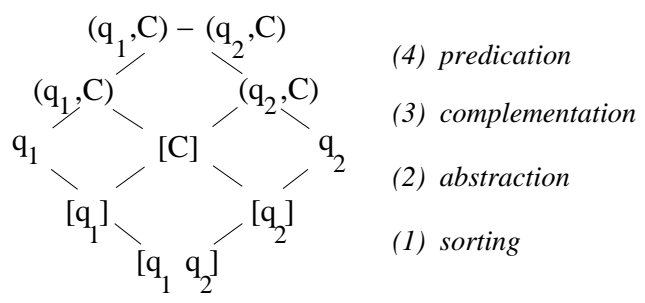

Figure 1: The processing schema of cognitive activity 


\subsection{Perception and cognition}

In this paper I assume that cognitive activity can be modeled by means of two processes that are isomorphic instances of the above schema. In the first process, perception, the 'goal' is the interpretation of a relation between the input qualia and the memory response representing the context (in this process the relation between the qualia themselves is secondary). The memory response arises due to the triggering input qualia. Depending on the activation of the memory, there may be qualia in the memory response having an intensity: (i) above or (ii) below threshold, respectively referring to an interpretation of the input which is in the brain's focus, and which is only complementary. A memory response of type (i) signifies the recognition of the input in the sense of agreement: the input is recognized or 'known' as such an entity. A type (ii) response refers to input recognition in the sense of possibility: the input is not recognized or 'not known', indicating that the memory response represents only a secondary or even less important aspect of the input qualia.

This way, the brain can distinguish between four different interpretations of the input stimulus: as a focused state $(A)$ and effect $(B)$, and a complementary state $(\neg A)$ and effect $(\neg B)$. Notice that ' $\neg$ ' is used as an indication of the denial of a positive identification, not as a Boolean operator. A definition of perception as an instance of the processing schema is omitted due to lack of space. A full account of such a model can be found in (Sarbo et al., 2006).

In the second process, cognition, the final signs generated by perception are interpreted as input qualia. In this process, the 'goal' is establishing a relation between the qualia themselves (now it is the relation between the input qualia and the context that is secondary). More specifically, the 'goal' of this process is a relation between the input qualia that are in the focus $(A, B)$, in the light of the qualia that are complementary $(\neg A, \neg B)$. In accordance with the current 'goal', the context ([C]) contains relational information about the input qualia.

The input signs appear as a 'primordial soup'. The important representation moment is step 3, in which, the input qualia are linked with background information (context). In conformity with the condition set for an interaction, there may be an interaction between $A$ and $\neg B$, and between $\neg A$ and $B$, but no interaction may occur between $A$ and $\neg A$, or $B$ and $\neg B$. This is because $A$ and $\neg A$ (but also $B$ and $\neg B$ ) arise due to the same input trigger, indicating that the two signs are not independent. The (re)cognition process is completed in step 4 , in which, a relation between
$A$ and $B$ is established. Cognition as a process is depicted in fig. 2.

The three relations, which correspond to the three types of interactions between the input qualia, can be characterized by means of the meaning of their constituents, as follows (an interaction, which is a relation, computationally, is denoted by a '-' symbol):

$A-\neg B$ :

$A$ is 'known', but $B$ is 'not known' (completion of the input state);

$B-\neg A$ :

$B$ is 'known', but $A$ is 'not known' (completion of the input effect);

\section{$(A, \neg B)-(B, \neg A)$ :}

both $A$ and $B$ are 'known' (establishment of the input relation).

If both $A$ and $B$ are 'not known', interpretation terminates, meaning that cognition, as a process, does not actually happen. Let me emphasize the mediation function of the context signs, in step 3 . Through the common meaning shared by the two signs, $\neg A$ and $\neg B$, the context implicitly determines the interpretation of the relation between $A$ and $B$. That relation can be called a 'proposition' which is a hypothesis ( $A$ 'is related to' $B$ or, briefly, $A$ ' $i s^{\prime} B$ ), but only if we agree that the input qualia are impossible to correct and that the relation 'generated' may represent only one of their aspects. The processing schema can be used recursively, by means of degenerately representing the final sign of the recognition process, as a quality. Recursive analysis can be necessary for the recognition of complex phenomena.

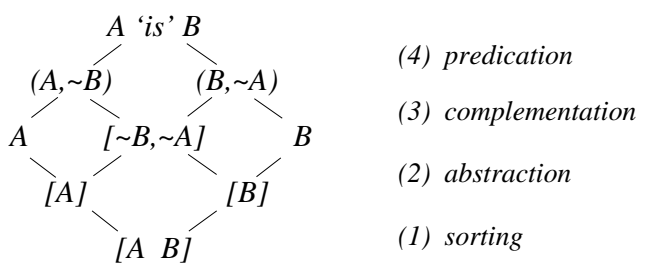

Figure 2: The signs generated by cognition as a process

\subsection{Logical analysis}

The interpretation of cognitive activity as the generation of three types of meaningful interactions illustrates the completeness of this process. This aspect becomes even more clear from the logical analysis of the processing schema. In this section I make an attempt to elaborate such an analysis, on the basis of the model of cognitive activity introduced in the previous 
section (the focus will be on cognition as a process; a similar analysis of perception can be made easily). First I associate a logical expression to each interpretation moment, on the basis of the common logical aspects exhibited by both of them. Second I introduce operations transforming those expressions to formal expressions generated by a Boolean logic. The hidden agenda of this section is a tacit introduction of logical concepts, in the process model of cognition. What makes this step especially important is that logical concepts have a precise meaning.

An essential element of a logical interpretation of cognitive activity as a process, is the abstraction of a common meaning of the two types of input qualia, which is the concept of a logical variable. In virtue of the duality of the input, the logical interpretation requires the introduction of two variables, which are denoted by $A$ and $B$. The collections of qualia that are in the focus, and that are complementary are represented, respectively, by means of a logical variable which is stated positively and negatively.

Perceived state and effect qualia that are in the focus are denoted, respectively, by $A$ and $B$; those that are complementary by $\neg A$ and $\neg B$. Notice the use of ' $\neg$ ' as logical negation, which is relative difference with respect to the collection of a type of qualia represented as a set. For example, the complementary subsets of the $A$-type qualia are denoted by $A$ and $\neg A$, ambiguously.

$\left[\mathrm{q}_{1}\right]=A+B,\left[\mathrm{q}_{2}\right]=A * B$ : The expression of the presence of the input qualia which are in the focus, as a meaningful co-existence $(A+B)$ and cooccurrence $(A * B)$, respectively, in the sense of possibility (' + ') and agreement ('*'). As $A$ and $B$ are commonly interpreted as logical variables, the separate representation of any one of the two types of input qualia contains a reference to both variables. The difference between the two types of relations between the input qualia is expressed by means of the difference between the two operators, ' + ' and ' $*$ '.

$\mathrm{q}_{1}=A * \neg B, \neg A * B$ : The logical abstraction of the input qualia which are in the focus as constituents, irrespective of the actually co-occurring other type of qualia. It is this perspective that makes the two signs synonymous (the "," in the definition of $q_{1}$ is a representation of this equivalence).

$\mathrm{q}_{2}=A * \neg B+\neg A * B$ : The expression of the input as an abstract event, defined by the possible coexistence or 'compatibility' of its abstract constituents (which are now interpreted differently). The context ([C]) is defined by the complementary qualia represented as a co-existence $(\neg A+\neg B)$ and a co-occurrence relation $(\neg A * \neg B)$. The synonymous representation of these signs is an expression of the secondary meaning of the context.

$\left(\mathrm{q}_{1}, \mathrm{C}\right)=A+\neg B, \neg A+B$ : The abstract constituents $\left(\mathrm{q}_{1}\right)$ completed with the context ([C]) or, alternatively, the actual meaning of the input qualia as constituents. For example, the meaning of $\neg A * B$ in context, is defined by the qualia complementing this abstract representation, which are: $A$ and $\neg B$. Alternatively, the actual meaning of $A$, as a constituent, is defined by $A$ itself and also by $\neg B$, the complementary qualia, linking $A$ with $B$ implicitly (as the relation between $A$ and $B$ is not yet realized, the qualia denoted by $B$ cannot contribute to this interpretation of $A$ ). As the two expressions of $A$, as an actual constituent, are related to each other by the relation of co-existence, the logical meaning of $\left(\mathrm{q}_{1}, \mathrm{C}\right)$ can be represented as $A+\neg B$. For the same reason, as in $\mathrm{q}_{1}$, the two representations of $\left(q_{1}, C\right)$ are interpreted as synonyms.

$\left(\mathrm{q}_{2}, \mathrm{C}\right)=A * B+\neg A * \neg B$ : The abstract compatibility relation in context, interpreting the input as a characteristic property appearing as an event. That event, which is a representation of the interaction between $A$ and $B$, is alternatively signified by the interaction between $\neg A$ and $\neg B$. The two signs signify the interaction which is in the focus, respectively, positively and negatively.

$\left(\mathrm{q}_{1}, \mathrm{C}\right)-\left(\mathrm{q}_{2}, \mathrm{C}\right)=A$ is $B$ : The expression of the logical relation between the input qualia which are in the focus, as a syllogistic proposition.

The above classification can be interpreted as a formal logic defined by a single operation, relative difference (' $\backslash$ '), which has three types: relative difference with respect to the type of quality itself (sorting); with respect to the other type of input qualia (abstraction); and, with respect to the input as a whole (complementation $)$. For example, $\left[\mathrm{q}_{2}\right]=A * B ; \mathrm{q}_{2}=\left[\mathrm{q}_{1}\right] \backslash\left[\mathrm{q}_{2}\right]$ : $(A+B) \backslash(A * B)=A * \neg B+\neg A * B ;\left(\mathrm{q}_{2}, \mathrm{C}\right)=\neg \mathrm{q}_{2}: \neg(A * \neg B$ $+\neg A * B)=A * B+\neg A * \neg B$. In the last example, relative difference with respect to the context ([C]) is interpreted as relative difference with respect to the universe (' 1 '), which explains the use of negation (' $\neg$ ') in the definition of $\left(q_{2}, C\right)$.

Notice in fig. 3 the presence of all Boolean relations on two variables, reinforcing our conjecture about the completeness of the cognitive process (' 0 ' and ' 1 ' can be defined as a representation of a 'notvalid' and a 'valid' input, respectively). The results of this analysis indicate that 'naive' logical signs hence also the concepts of cognitive activity, as a process, can be defined as an interaction (relation) between neighboring signs. In fig. 3 , such signs are connected 


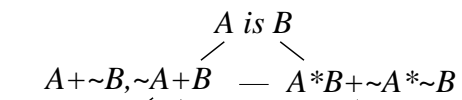

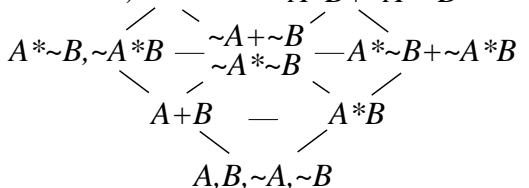

Figure 3: Logical interpretation of cognitive activity

with a horizontal line. In a computational interpretation, the combinatory properties of the signs can be represented by finite sets, and the interactions between signs by Boolean operations on sets. A full account of 'naive' logic can be found in (Sarbo and Farkas, 2001).

The processing schema can be used recursively, in which case the final sign generated by one process can be subject to sorting by a subsequent next process. That $\left(q_{1}, C\right)-\left(q_{2}, C\right)$ is suited for this operation can be justified by the logical analysis of the constituents of predication. This reveals that $\left(\mathrm{q}_{1}, \mathrm{C}\right)$ and $\left(\mathrm{q}_{2}, \mathrm{C}\right)$ can be interpreted, respectively, as a co-existence ('+'), and a co-occurrence relation (' $*$ ') defined on same terms:

$$
\left(\mathrm{q}_{1}, \mathrm{C}\right)=(A+\neg B, \neg A+B)
$$$$
\left(\mathrm{q}_{2}, \mathrm{C}\right)=(A+\neg B) *(\neg A+B)
$$

Notice that the above expression of $\left(\mathrm{q}_{2}, \mathrm{C}\right)$ is an alternative form for its logical representation as $(A * B)+(\neg A * \neg B)$. In logic, recursion can be syntactically represented by means of nesting (parenthesis).

\subsection{Naive versus Boolean logic}

In virtue of its relation with cognitive activity, 'naive' logic can be more powerful than Boolean logic. For example, the synonymous interpretation of $\mathrm{q}_{1}$, as a constituent state and event, enables the corresponding logical expressions, $A * \neg B$ and $\neg A * B$, to be used interchangeably.

This dexterity of 'naive' logic is opposed to the efficiency of Boolean logic. By refraining from the synonymous representation of 'naive' logical signs and defining a suitable calculus, Boolean logic introduces an interpretation that can be computationally more efficient. While 'naive' logic may only establish a relation between two variables at a time, and the order of logical computations is dictated by the processing schema, Boolean logic may combine variables and compute operations in any order. A final difference between 'naive' and Boolean logic is due to the different interpretation of the logical values 'true' and 'false'. In Boolean logic those values are defined as constants. Not in 'naive' logic, in which 'true' and 'false' are interpreted as a representation of the status of the sign recognition process, that can be 'valid' or 'not valid'.

Can 'naive' logic be useful in practice? No, if we only use the computer for doing calculations. Yes, if such calculations have to be communicated with the human user. Following the theory of this paper, the formal computations by the computer can be presented as the signs of a recognition process of some phenomenon. The conjecture of this research is that such a presentation may enable the human user to more directly conceptualize information generated by the computer, as knowledge. A phenomenon, similar to this one, is apparent motion perception, in which, pictures presented in a certain way are experienced as motion. Individual pictures representing static information correspond to a percept; the experience of a series of pictures as motion, to a meaningful interpretation of subsequent percepts.

\section{A Peircean semiotic interpretation}

That the intuitive and the formal logical interpretation of a sign are tightly related to each other must be clear from our earlier explanation of the logical relations of cognition. This dependency forms the basis for the semiotic interpretation of the nine types of logical relations and interpretation moments. For example, $\left[\begin{array}{ll}q_{1} & q_{2}\end{array}\right]$ represents that the aspect of quality is present in the appearance of the input as a 'primordial soup'; $\left[\mathrm{q}_{2}\right]$ represents that the aspect of simultaneity is a primary element of the input, as an appearance (event) that happens now; $q_{2}$ represents that the compatibility of the abstract meaning of the input qualia is expressive of a rule-like relation. A complete overview of the meaning aspects exhibited by the logical relations as well as the interpretation moments of the processing schema is given in fig. 4 .

From this semiotic interpretation of the logical relations, the analogy with Peirce's nine signs, as meaning aspects, follows trivially. For example, $\left[q_{1} q_{2}\right]$, [ $\left.q_{2}\right]$, and $\mathrm{q}_{2}$, this time interpreted as proto-signs, ${ }^{1}$ corresponds to the Peircean concept of a qualisign, sinsign, and legisign, respectively (Sarbo, 2006). The classification of Peirce's nine signs is depicted in fig. 5.

In summary, the logical interpretation of the processing schema opens the way to a Peircean semiotic account of our model, revealing the existence of a relation between the interpretation moments, and the nine kinds of aspects or parameters of (full) meaning, defined by Ch.S. Peirce (Peirce, 1931).

\footnotetext{
${ }^{1}$ Proto-signs are in a process of becoming a sign.
} 


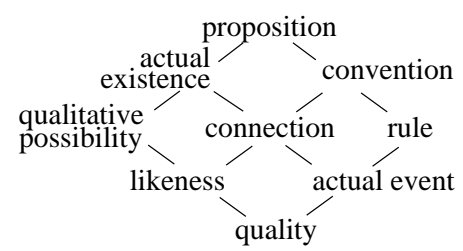

Figure 4: The semiotic interpretation of logical signs and interpretation moments

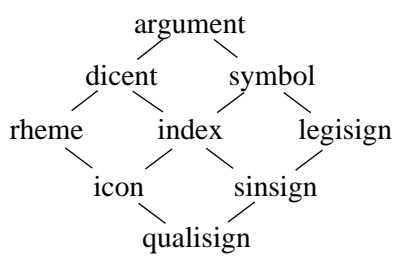

Figure 5: Peirce's nonadic classification of signs

\section{Summary and further research}

In past research we applied our process model of cognitive activity for knowledge representation in different domains, such as, natural language, 'naive' reasoning and mathematics (the conception of number). We have shown that the processing schema is also suited for a computational interpretation. The combinatory properties of signs can be represented by finite sets, and interactions between signs by Boolean operations on sets. The resulting formal model can be proved to be linearly complex in the number of input qualia (Sarbo and Farkas, 2002).

In virtue of its importance for cognitive activity, 'naive' logic can be practical for the development of user interfaces and ontologies (Sarbo et al., 2006). Most recently we applied the theory for requirement engineering, in particular in the initial conceptualization phase of problem specification (van Breemen et al., 2007).

While the focus of past research has been on a justification of the theory, by means of testing it in different domains of human knowledge, current research is centered around the embedding of the process model of cognitive activity in the Peircean theory of interpretants (van Breemen and Sarbo, 2007).

\section{REFERENCES}

Bergman, M. (2000). Reflections on the role of the communicative sign in semeiotic. Transactions of the Charles S. Peirce Society: A
Quarterly Journal in American Philosophy, XXXVI(2):225-254.

Farkas, J. and Sarbo, J. (2000). A Logical Ontology. In Stumme, G., editor, Working with Conceptual Structures: Contributions to ICCS'2000, pages 138-151, Darmstadt (Germany). Shaker Verlag.

Gazzaniga, M. (1998). Cognitive neuroscience. W.W. Norton, New York, London.

Harnad, S. (1987). Categorical perception: the groundwork of cognition. Cambridge University Press, Cambridge.

Kalat, J. (2004). Biological psychology. Wadsworth/Thomson Learning, Belmont, CA.

McCarthy, J. and Hayes, P. (1969). Some philosophical problems from the standpoint of artificial intelligence. In Meltzer, B. and Michie, D., editors, Machine Intelligence 4, pages 463-502. Edinburgh University Press.

Peirce, C. (1931). Collected Papers of Charles Sanders Peirce. Harvard University Press, Cambridge.

Sarbo, J. (2006). Peircean proto-signs. In Dubois, D. M., editor, AIP Conference Proceedings, volume 839, pages 474-479, Liege, Belgium. Best Paper Award.

Sarbo, J. and Farkas, J. (2001). A Peircean ontology of language. In Delugach, H. and Stumme, G., editors, ICCS'2001, volume 2120 of LNAI, pages 1-14, Stanford (CA). Springer-Verlag.

Sarbo, J. and Farkas, J. (2002). A linearly complex model for knowledge representation. In U.Priss and Corbett, D., editors, Conceptual Structures: Integration and Interfaces (ICCS'2002), volume 2193 of LNAI, pages 20-33, Borovets (Bulgaria). Springer-Verlag.

Sarbo, J., Farkas, J., and van Breemen, A. (2006). Natural Grammar. In Gudwin, R. and Queiroz, J., editors, Semiotics and Intelligent System Development, pages 152-175, Hersey (PA). Idea Group Publishing.

van Breemen, A. and Sarbo, J. (2007). Sign processes and the sheets of semeiosis $\left(\mathrm{S}_{S}\right)$. Technical Report ICIS-R07003, Radboud University, Nijmegen, The Netherlands.

van Breemen, A., Sarbo, J., and van der Weide, T. P. (2007). Toward a theory of natural conceptualization. Technical Report R07002, University of Nijmegen. 